\title{
Channel Coding Optimization Based on Slice Visibility for Transmission of Compressed Video over OFDM Channels
}

\author{
Laura Toni, Member, IEEE, Pamela C. Cosman, Fellow, IEEE, and Laurence B. Milstein, Fellow, IEEE
}

\begin{abstract}
Optimization of multimedia transmissions over wireless channels should be aimed at maximizing the video quality perceived by the final user. For transmission of video sequences over an orthogonal frequency division multiplexing (OFDM) system in a slowly varying Rayleigh faded environment, we develop a cross-layer technique, based on a slice loss visibility (SLV) model used to evaluate the visual importance of each slice. In particular, taking into account the visibility scores available from the bitstream, depending on the scenario, we optimize the mapping of video slices within a 2-D time-frequency resource block and/or the channel code rates, in order to better protect more visually important slices. The proposed algorithm is investigated for several scenarios, with different levels of information about the channel available in the optimization process. Results demonstrate that, for different physical environments and different video sequences, the proposed algorithm outperforms baseline ones which do not take into account either the SLV or the CSI in the video transmission.
\end{abstract}

Index Terms-Slice loss visibility, channel coding, cross-layer design, diversity, multimedia communications, orthogonal frequency division multiplexing (OFDM).

\section{INTRODUCTION}

$\mathbf{S}$ INCE cross-layer optimization schemes can improve the quality of experience (QoE) by optimizing the network architectures across traditional OSI stack layers, such techniques have been under intense research as primary strategies for adaptation to dynamic channel conditions [1]-[3]. A wellperforming cross-layer optimization design strongly depends on an efficient QoE metric, that faithfully reflects the level of quality experienced by the user [4]. This has led researchers to investigate objective metrics able to assess the visual quality of wireless video communication [5]. One approach is the evaluation of a set of simple metrics which can provide a level of priority of the encoded slices to be used in the optimization problems. When fine-grain scalable video sequences are considered, each bit of the encoded enhancement bitstream within a frame is more important than the subsequent bit,

Manuscript received 7 August 2011; revised 20 January 2012. Part of this work has been presented at the Workshop on Acoustics and Video Coding and Communication (AVCC) at the 2011 IEEE Intl. Conf. on Multimedia \& Expo (ICME). This research was supported in part by the Intel-Cisco Video Aware Wireless Networks (VAWN) program, by the office of Naval Research under Grant N00014-11-1-0733, and by the UCSD FWGrid Project, NSF Research Infrastructure Grant Number EIA- 0303622.

L. Toni, P. C. Cosman and L. B. Milstein are with the Department of Electrical and Computer Engineering, University of California at San Diego, La Jolla, CA 92093-0407 USA (e-mail: \{ltoni,pcosman\}@eng.ucsd.edu; milstein@ece.ucsd.edu).

Digital Object Identifier 10.1109/JSAC.2012.120803. and the level of priority is intrinsic in the encoding process. By adopting unequal error protection (UEP), a more reliable transmission is offered to the more important bits, and the QoE can be improved compared with an equal error protection (EEP) system [6]-[8].

For non-scalable video sequences, assigning priority levels to portions of the compressed bitstream is more challenging, as it is not the simple case that earlier bits are more important than later ones. In [9], the authors optimized a H.264 flexible macroblock ordering (FMO)-based classification of the macroblocks with a jointly optimal channel rate allocation. The final goal was to exploit and improve the error resilience features of an H.264/AVC codec, when video sequences were transmitted over erasure channels. Rather than operating at the source encoder, in [10], rate-compatible punctured convolutional (RCPC) code rate allocation was optimized for non-scalable pre-encoded video sequences transmitted over error-prone channels. The authors proposed a low-complexity algorithm, which optimized the RCPC rate per packet using the subgradient method to search in the dual domain, minimizing the distortion of the video sequence transmitted over additive white Gaussian noise (AWGN) channels. In both these works, the mean square error (MSE) induced by a packet loss was considered as the metric for the cross-layer optimization. As in [10], we aim at optimizing the RCPC channel code rates for non-scalably encoded video sequences. Our goal is to propose a cross-layer technique to optimize the QoE metric, without any change to the source encoder.

When dealing with QoE metrics, an important aspect is the relation between the distortion metric and the packet losses [11], [12]. This relation usually depends on many parameters (e.g., the coding scheme, the bit rates and the network architectures), which are not always included in distortion metrics. A great part of the effort to understand the visual impact of packet losses has been focused on modeling the mean quality of videos as a function of average packet loss rate (PLR) [13]-[15]. However, PLR can provide wrong interpretations of video quality since packet losses are perceptually not equal. In [16], [17], the authors studied the problem of predicting packet loss visibility for non-scalable compressed video, and they proposed a metric able to predict the probability that a slice loss is visible by a final user. The proposed slice loss visibility (SLV) score can be viewed as priority level information for non-scalably encoded video slices, which can be employed in cross-layer optimization techniques. In [17], the SLV model 
was used to design a policy for perceptual-quality based packet discarding. An intermediate router in a congested network, for example, might employ the SLV metric to decide which packets should be dropped to minimize degradation in the quality of the transmitted video streams.

In this paper, we aim at maximizing the QoE of a nonscalable bitstream when the compressed video sequence is transmitted over a frequency selective orthogonal frequency division multiplexing (OFDM) network. Based on the information available from the feedback channel and the application layer, we propose a technique that jointly groups the encoded bitstream into packets, optimizes the channel code rate for each packet, and maps the encoded slices into the 2-D resource block (RB). The proposed algorithm can be applied to a multitude of scenarios, from point-to-point communication, in which both channel state information (CSI) and SLV information are available at the transmitter, to a broadcasting scenario, in which the CSI will not be available in the feedback channel. At the same time, based on the SLV and the physical environment, the cross-layer algorithm might select a UEP or an EEP profile as the best choice.

The main goals of this work are the following:

i) to demonstrate that the SLV model can improve the system design and optimization;

ii) to provide a study from which a system designer is able to select the best mapping and forward error correction (FEC) profile based on the considered scenario;

iii) to examine the performance gain of three algorithms that have increasing levels of complexity.

The remainder of this paper is organized as follows. In Section II, we describe some technical preliminaries, including the basics of SLV and OFDM systems. In Section III, we discuss the proposed cross-layer diversity approach, and the associated tradeoff issues. The theoretical problem formulation is described in Section IV. In Section V, we provide simulation results and discussion, and we conclude in Section VI.

\section{Preliminaries}

In the following, we provide a brief introduction to the SLV metric and a description of the system model.

\section{A. Slice Loss Visibility Overview}

We consider a non-scalable video encoder and assume that each frame is divided into $N_{\mathrm{s}}$ slices (each slice consists of a constant number of macroblocks), as depicted in Fig. 1. The priority level of each slice is determined by the SLV model which estimates the quality degradation the video experiences when that slice is lost. The SLV model was introduced in [17] as a bitstream-based metric for non-scalable compressed video. Bitstream-based metrics predict video quality using packet header information and limited information from the encoded bitstream, such as motion vectors, but do not involve a full decoding or pixel-level reconstruction of the video source. The authors conducted subjective tests in which the viewers' task is to indicate when they observe a packet loss artifact. From these tests, an SLV metric was proposed with the goal of predicting whether a packet loss in the video stream is visible to a viewer. Note that, in [17], one of the factors in the packet frame \#k

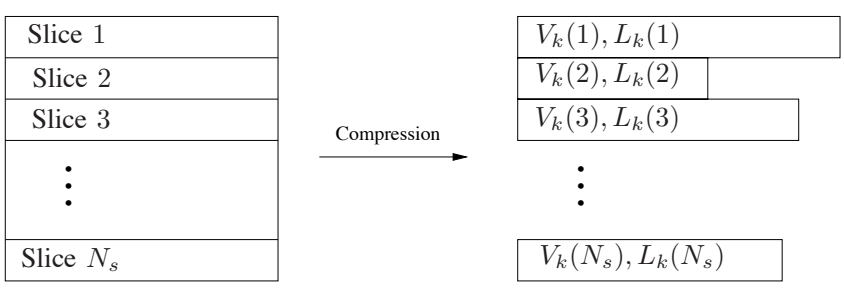

Fig. 1. Slice structure for the $k$ th frame of a video sequence.

loss visibility model is called "SpatialExtent" and it refers to the spatial extent of the loss caused by the loss of this packet. In our work, we use the visibility model to give us a score for one slice at a time (we define a slice as one horizontal row of macroblocks), in which case this factor is identical for all slices, and the packet loss visibility model of [17] becomes our SLV model. The proposed SLV scores provide priority level information for non-scalably encoded video slices, which we will employ in our cross-layer optimization techniques.

In our model, the $i$ th slice of frame $k$ is encoded into $L_{k}(i)$ bits and has a priority level $V_{k}(i)$. The $V_{k}(i)$ values range from 0 to 1 , where $V_{k}(i)=0$ means that the slice, if lost, would produce a loss artifact glitch that would likely not be noticed by any observer, and $V_{k}(i)=1$ means that the loss artifact would likely be seen by all users. So, each encoded slice is characterized by the pair $\left(V_{k}(i), L_{k}(i)\right)$, for $i=1, \ldots, N_{\mathrm{s}}$ and $k=1, \ldots, N_{\mathrm{F}}$, where $N_{\mathrm{F}}$ is the number of frames per group of pictures (GOP).

\section{B. System Model}

The video sequences are transmitted over frequencyselective OFDM channels, and we use a block fading channel model to simulate the frequency selectivity [18]. In this model, the spectrum is divided into blocks of size $(\Delta f)_{\mathrm{c}}$. Subcarriers in different blocks are assumed to fade independently; subcarriers in the same block experience identical fades. As illustrated in Fig. 2, we assume an OFDM system with an overall system bandwidth $W_{\mathrm{T}}$, such that we can define $N$ independent subbands. Each subband consists of $M$ correlated subcarriers spanning a total bandwidth of $(\Delta f)_{\mathrm{c}}$. The total number of subcarriers in the OFDM system is $N_{\mathrm{t}}=N \times M$. Often, the maximum achievable frequency diversity $\mathcal{D}_{\mathrm{f}}$ is given by the ratio between the overall system bandwidth $W_{\mathrm{T}}$ and the coherence bandwidth $(\Delta f)_{\mathrm{c}}$.

In the time domain, the channel experiences slow fading, so that a constant fade per packet is considered. Although there is no time diversity to exploit by using channel codes, coding gain can still be obtained, and a concatenation of cyclic redundancy check (CRC) codes and RCPC codes are applied to each transmitted packet. We assume that the fading gain $h_{i}$ experienced by the $i$ th subcarrier is distributed as a complex Gaussian random variable with mean zero and variance $\sigma_{h}^{2}$ per dimension. We denote the instantaneous signalto-noise ratio (SNR) experienced by the $i$ th subcarrier by $\gamma_{i}=\left|h_{i}\right|^{2} E_{\mathrm{s}} / N_{0}, i=1, \ldots, N_{\mathrm{t}}$, which will be constant over the whole subband (i.e., $\gamma_{i}=\gamma_{j}$ if the $i$ th and $j$ th subcarriers are within the same subband). Note that $E_{\mathrm{s}}$ is the transmitted 


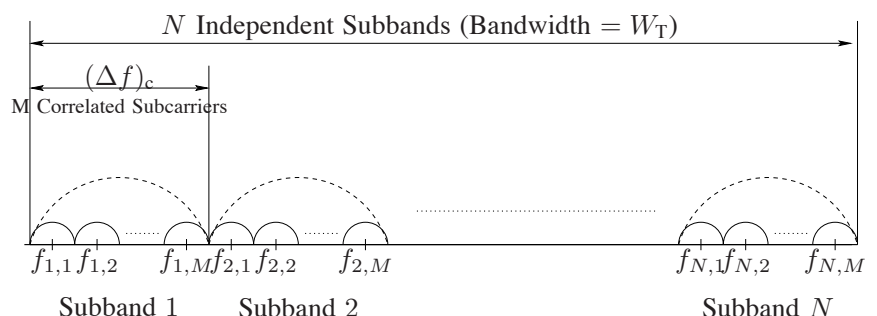

Fig. 2. OFDM spectrum.

symbol energy, and $N_{0} / 2$ is the two-sided spectral density of the AWGN. The RB will experience a constant mean SNR, which is denoted by $\bar{\gamma}=\mathbb{E}\left\{|h|^{2}\right\} E_{\mathrm{s}} / N_{0}=2 \sigma_{h}^{2} E_{\mathrm{s}} / N_{0}$, where $\mathbb{E}\{\cdot\}$ denotes the statistical expectation (evaluated over the fading).

\section{Scenarios Considered}

We now describe the possible scenarios considered in our work and listed in Table I. In particular, we focus on the availability of instantaneous CSI and SLV parameters. We consider all possible combinations of knowing the instantaneous CSI and not the SLV, knowing the SLV and not the instantaneous CSI, knowing both pieces of information, or knowing neither. If one knows neither (Table line 0 ), the algorithm corresponds to our baseline approaches (sequential and random) which are described later.

In Fig. 3, a schematic description of the proposed algorithm is depicted to show how the SLV and CSI information might be employed in the optimization scheme. While a detailed characterization of the cross-layer algorithm is provided in the next section, the main point is that if the sender has at least one of the two types of information, then the algorithm can exploit the information. In particular, we consider two types of exploitation: the first is UEP FEC (using different RCPC channel code rates for different slices, or for different subcarriers) and the second is slice-to-subcarrier mapping, in which the algorithm maps the visually more important slices to the better subcarriers. Note that the UEP FEC could, in principle, make use of the information of either the SLV or the instantaneous CSI, or both. That is, heavier error protection could be provided to specific slices (because they are more important) or to specific subcarriers (because they are not reliable). In contrast, the slice-to-subcarrier mapping operation requires both the SLV and instantaneous CSI information. If the instantaneous CSI is available from the feedback channel, the subcarriers of the RB can be ordered from the most reliable to the least reliable, and if in addition the SLV information is available, then the most important slices (highest SLV parameter) can be allocated (mapped) to the most reliable subcarriers.

So, lines 2, 3, 5 and 6 in Table I are marked as impossible because the slice-to-subcarrier mapping can only be done if you know both pieces of information. Line 1 of the table is skipped as we are not interested in the case where the SLV is not known. Line 7 of the table is skipped because, knowing both pieces of information, it seems more sensible to use the information for mapping (Line 8) or for both UEP and mapping (Line 9). Accordingly, this paper considers the scenarios corresponding to lines 4,8 , and 9 of the table, which are referred to as Scenarios $\boldsymbol{A}, \boldsymbol{B}$, and $\boldsymbol{C}$, respectively, in addition to the two baseline algorithms in line 0 .

Scenario $\boldsymbol{A}$ covers channels such as a broadcast and/or fast fading, where instantaneous CSI is not available. This means that the "Subcarrier Ordering" step in Fig. 3 of the optimization algorithm cannot be done. However, the SLV information is known, and provides priority levels to the slices that should be mapped in the RB. So we use UEP to ensure that the most visible slices (i.e., slices which, if lost, are most likely to produce a visible glitch) will be transmitted over subcarriers protected with the lowest RCPC code rate.

In Scenarios $\boldsymbol{B}$ and $\boldsymbol{C}$, we would like to see the gain of a cross-layer optimization when both the SLV and the CSI are available at the transmitter. This would be the case for point-to-point communications. In both Scenarios $\boldsymbol{B}$ and $\boldsymbol{C}$, instantaneous CSI is exploited to order subcarriers from best to worst. Scenario $\boldsymbol{B}$ is the lower complexity algorithm, where the more important slices (in order) are allocated to better subcarriers (in order). To keep the algorithm simple, we do not consider UEP; all subcarriers have the same RCPC code rate. In Scenario $\boldsymbol{C}$, at the cost of increasing optimization complexity, we investigate a joint UEP profile-slice mapping optimization.

\section{VQM versus $S L V$}

The Video Quality Metric (VQM) is a standardized fullreference method of objectively measuring video quality considering both coding artifacts and transmission errors [19]. It measures the perceptual effects of a broad range of quality impairments, including blurring, jerky or unnatural motion, global noise, block distortion, color distortion and packet loss. It has been adopted by the ANSI as a U.S. national standard and as an international ITU Recommendation and has been shown to be better correlated with human perception than other full reference video quality metrics. The output scores range from 0 (best quality) to 1 (worst quality). In this work we use VQM to evaluate the quality of our output video sequences.

Evaluating the average VQM score for a particular encoding configuration requires passing the encoded video over many simulated realizations of the channel, decoding the video, and putting the resulting video, together with the original, into the VQM metric calculation. As a design algorithm to choose the best encoding configuration, this evaluation with multiple realizations of the channel is time consuming. So we also consider an approach in which the algorithm design involves optimizing a weighted SLV score (which can be done numerically, without channel realizations and decoding operations) rather than optimizing for the VQM. However, it is important to note that, even when the design of the encoding configuration is based on weighted SLV, the final performance evaluation is always based on VQM.

\section{Design Algorithm based on Optimizing VQM}

The main steps of the proposed algorithm are applied to each GOP of the video sequences. Since the number of bits in which a single frame is encoded might be considerably 
TABLE I

INVESTIGATED SCENARIOS AND ASSOCIATED OPTIMIZATION SCHEMES.

\begin{tabular}{|c|c|c|c|c|c|}
\hline & \multicolumn{2}{|c|}{ Available Information } & \multicolumn{2}{c|}{ Algorithm includes } & \\
\hline & instantaneous CSI & SLV & FEC-UEP & $\begin{array}{c}\text { Slice/Subcarrier } \\
\text { Mapping }\end{array}$ & \\
\hline 0 & & & & & Baselines: sequential, random \\
\hline 1 & $\mathrm{X}$ & & $\mathrm{X}$ & & Skip \\
\hline 2 & $\mathrm{X}$ & & & $\mathrm{X}$ & impossible \\
\hline 3 & $\mathrm{X}$ & & $\mathrm{X}$ & $\mathrm{X}$ & Scenario $\boldsymbol{A}$ \\
\hline 4 & & $\mathrm{X}$ & $\mathrm{X}$ & & impossible \\
\hline 5 & & $\mathrm{X}$ & & $\mathrm{X}$ & impossible \\
\hline 6 & & $\mathrm{X}$ & $\mathrm{X}$ & $\mathrm{X}$ & Skip \\
\hline 7 & $\mathrm{X}$ & $\mathrm{X}$ & $\mathrm{X}$ & & Scenario $\boldsymbol{B}$ \\
\hline 8 & $\mathrm{X}$ & $\mathrm{X}$ & & $\mathrm{X}$ & Scenario $\boldsymbol{C}$ \\
\hline 9 & $\mathrm{X}$ & $\mathrm{X}$ & $\mathrm{X}$ & $\mathrm{X}$ & \\
\hline
\end{tabular}

different (e.g., the number of bits for an I-frame will be greater than the number required for a B-frame), assuming a constant $\mathrm{RB}$ for each frame would not make good use of available resources. Instead, we adopt a fixed-sized 2D time-frequency RB for each GOP. This cross-layer choice corresponds to a very common approach in application-layer video rate control, in which the number of bits allocated to individual frames is allowed to vary, but the number of bits given to each GOP is held roughly constant.

As illustrated in Fig. 3, the current GOP is processed by a joint mapping/coding step. In our notation, $N_{\mathrm{F}}$ frames form a GOP; each frame is divided into $N_{\mathrm{s}}$ slices. After the optimization algorithm, groups of slices are allocated to packets. Then, each packet will consist of one or more slices plus the FEC added by the RCPC code and the bits added by the CRC code. It should be noted that information bits and CRC/RCPC parity symbols would be interleaved in an actual system. However, for illustration, we show the de-interleaved version so that the relative amounts of parity symbols and information symbols can be depicted. After channel coding, packets have constant length (equal to $L_{\mathrm{m}}$ modulated symbols) and will be assigned to a subcarrier. Then, for each RB, $N_{\mathrm{t}}$ packets will be transmitted on $N_{\mathrm{t}}$ subcarriers.

Note that the mapping of the slices into the RB and the channel code rate optimization are mutually dependent processes. The best FEC profile for the RB depends on the SLV parameters for the slices within each packet. On the other hand, the mean SLV for a packet depends on how many information bits get allocated to the packet, and thus it depends on the channel code rates adopted for the RB. This joint allocation/coding step is the focus of our work. We propose an algorithm able to allocate the slices of each GOP and evaluate the optimal RCPC profile by taking into account the SLV, the channel model parameters, and either the frequency diversity order (Scenario $\boldsymbol{A}$ ) or the instantaneous CSI (Scenarios $\boldsymbol{B}$ and $\boldsymbol{C}$ ). The proposed method can be described with the following steps.

\section{Step 1: Slice Ordering and Grouping}

We order all the $N_{\mathrm{F}} \times N_{\mathrm{S}}$ slices of the GOP and divide them into $K_{v}$ groups based on the SLV parameter. The first group $\left(\Lambda_{1}\right)$ contains the most visible slices and the last one $\left(\Lambda_{K_{v}}\right)$ contains the least visible slices. Equally spaced thresholds in the range $[0,1]$ are chosen to divide the slices into $K_{v}$ groups.
Note that the slice grouping will be applied in both Scenarios $\boldsymbol{A}$ and $\boldsymbol{C}$, while Scenario $\boldsymbol{B}$ might be considered as a degenerate case in which $K_{v}=1$, and Step 1 reduces to slice ordering.

\section{Step 2: Subcarrier Ordering}

Information about the fading gain, if available, is exploited in this step. The subcarriers of the 2-D RB are ordered from the most reliable to the least reliable. Denoting by $\gamma=\left[\gamma_{1}, \gamma_{2}, \ldots, \gamma_{N_{\mathrm{t}}}\right]$ the SNR information available in the feedback channel (Scenarios $\boldsymbol{B}$ and $\boldsymbol{C}$ ), the subcarriers are ordered in such a way that $\gamma_{\mathrm{sort}, 1} \geq \gamma_{\mathrm{sort}, 2} \geq \ldots \geq \gamma_{\mathrm{sort}, N_{\mathrm{t}}}$, where $\gamma_{\text {sort }}=\left[\gamma_{\text {sort }, 1}, \ldots, \gamma_{\text {sort }, N_{\mathrm{t}}}\right]$ is the ordered SNR vector. When Scenario $\boldsymbol{A}$ is considered, since no instantaneous CSI is available at the transmitter, the subcarriers are not ordered.

\section{Step 3: Mapping and RCPC code rate optimization}

Slices within each group are given the same protection; we assign a single RCPC code rate for each group. We seek the best rate vector $r^{*}=\left[r_{1}, r_{2}, \ldots, r_{K_{v}}\right]$, where $r_{j}$ denotes the RCPC code rate assigned to the slices within group $\Lambda_{j}$. That is, all slices in the $j$ th visibility group will be allocated to packets encoded with a code rate $r_{j}$. We use $R_{i}$ to denote the RCPC code rate for the $i$ th subcarrier or packet. ${ }^{1}$ As depicted in Fig. 4, where Step 3 is described in detail, if the $i$ th packet contains slices from group $\Lambda_{j}$, then $R_{i}=r_{j}$.

Step 3 involves repeatedly cycling through the following three phases:

a) Among all the possible FEC profiles, select a rate vector $r$ to be evaluated.

b) Based on both the information about the channel (either instantaneous CSI or frequency diversity order, depending on the scenario) and $r$, the slices of each group will be allocated to subbands. For example, assuming that the group $\Lambda_{1}$ needs to be allocated to the RB, the first $m$ subcarriers will be occupied by the group $\Lambda_{1}$, and each one of these $m$ packets will be protected with an RCPC code rate $R_{i}=r_{1}$, for $i=1, \ldots, m$, plus the CRC code. The number of subcarriers in which group

\footnotetext{
${ }^{1}$ Since the CRC code will assign a constant number of bits per subcarrier, from here onwards, in the optimization description we will only refer to the $\mathrm{RCPC}$ channel code rate.
} 
SLV of the current GOP

(Scenarios $\boldsymbol{A}, \boldsymbol{B}$, and $\boldsymbol{C}$ )

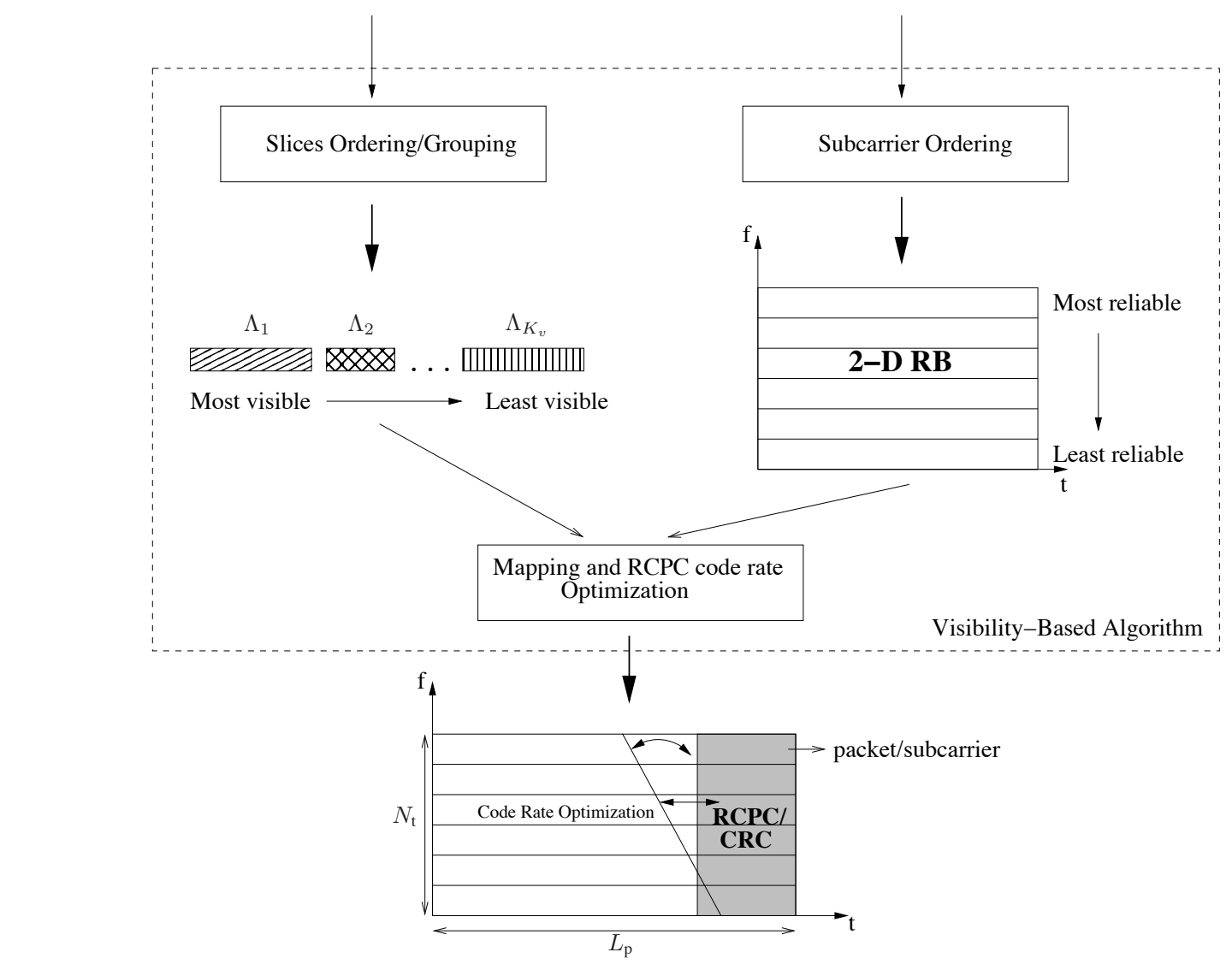

CSI

(Scenarios $\boldsymbol{B}$ and $\boldsymbol{C}$ )

Fig. 3. Slice ordering and subcarrier mapping. Note that the CRC/RCPC parity symbols are interleaved with the information symbols in an actual system.

$\Lambda_{1}$ is allocated has to meet the following constraint

$$
\sum_{k: V_{k} \in \Lambda_{1}} L_{k}(i) \leq m \times L_{\mathrm{p}} \times r_{1}
$$

where $L_{\mathrm{p}}=L_{\mathrm{m}} \log _{2}(\mathrm{Mod})$, where Mod is the constellation size of the adopted modulation (QPSK in our case, constant for all the RBs), and $L_{\mathrm{p}} \times r_{1}$ is the number of information bits per subcarrier. The first group will be allocated to the first $m$ subcarriers, and the other groups will be sequentially allocated within the RB. Note that for Scenarios $\boldsymbol{B}$ and $\boldsymbol{C}$, this allocation choice allows us to map the most important slices to the most reliable channels. For the case in which instantaneous CSI is not available (Scenario $\boldsymbol{A}$ ), we use simply a sequential mapping. This step is considered for all $K_{v}$ groups. It is worth noting that the visibility model is applied to one slice (one horizontal row of macroblocks) at a time. The slice is then allocated to a position in the resource block. Wherever possible, we try to allocate the entire slice in a given subcarrier, but if it has too many bits, then it may spill over to another subcarrier. In particular, if the first part of the slice is allocated in the $j$ th subcarrier, the remaining bits of the slice will be allocated in the next subcarrier, i.e., the $(j+1)$ th one in the SNR vector $\gamma$ for Scenario $\boldsymbol{A}$ and the $(j+1)$ th one in the ordered SNR vector $\gamma_{\text {sort }}$ for Scenarios $\boldsymbol{B}$ and $\boldsymbol{C}$. Moreover, if the number of bits in the GOP is greater than the number of information bits available in the RB, randomly chosen slices from the least important group are dropped. We use the word "dropped" or "discarded" to describe slices which are preemptively dropped by the encoder because the RCPC code rate profile does not allow all the information bits to fit in the $\mathrm{RB}$, whereas we use the word "lost" to describe slices which are allocated to the RB but which fail to be decoded correctly at the receiver. Both dropped and lost slices are concealed at the decoder.

c) Once the slices are allocated within the RB, based on the chosen FEC profile $r$, we evaluate the mean VQM score by simulating 1000 random realizations of the channel. The mean VQM score is then compared with the best one, $\mathrm{VQM}_{\text {best }}{ }^{2}$ If $\operatorname{VQM}(\boldsymbol{r})<\mathrm{VQM}_{\text {best }}$, then the best VQM is updated.

The phases $a, b$, and $c$ are repeated for all the possible FEC profiles considered in the optimization process. With this algorithm, the best FEC profile is chosen for each GOP of the video sequence (GOP-by-GOP optimization). Alternatively, if the final goal of the proposed method is to choose the RCPC channel code rate profile able to maximize the mean quality of the whole video sequence, Step $\mathbf{1}$ and Step $\mathbf{2}$ are repeated for all the GOPs of the sequence. Then, for each $r$ in Step $\mathbf{3} a$, each GOP is mapped into a RB (Step $\mathbf{3} b$ ), and then, the VQM is evaluated for the whole video sequence (Step 3c). It is worth noting that using the GOP-by-GOP optimization rather

\footnotetext{
${ }^{2} \mathrm{As}$ an initial value, we set $\mathrm{VQM}_{\text {best }}=1$.
} 


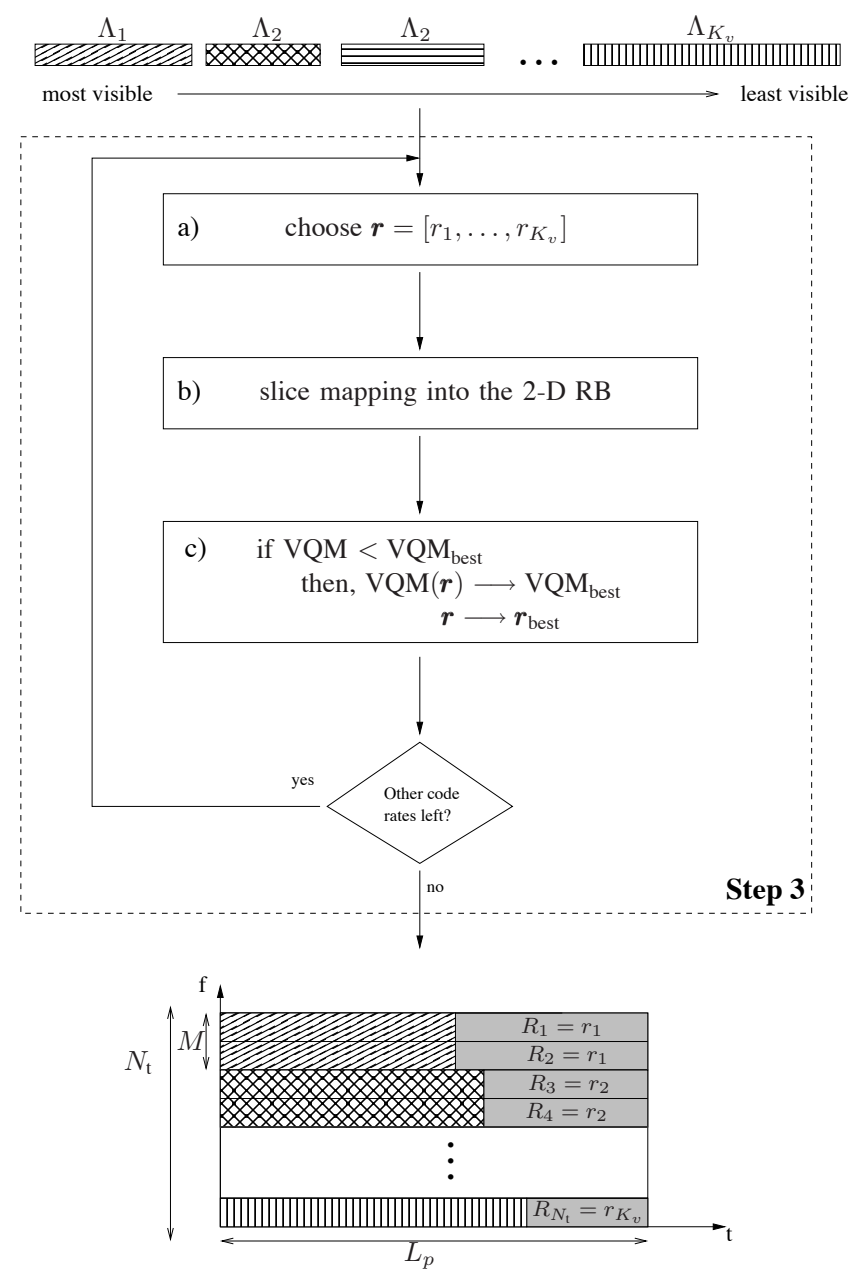

Fig. 4. Step 3: Mapping and RCPC code rate optimization.

than the whole video sequence optimization, one increases the algorithm complexity but substantially decreases the latency and increases the performance.

\section{Optimization OF SLV SCORE}

In the previous section, we considered an optimization aimed at minimizing the VQM score. This implies that, for each RCPC profile, we need to simulate many realizations of the RB transmission, decode the received video sequences, and evaluate the average quality of the received bitstream in terms of VQM. A simpler optimization would be one in which the best RCPC code rate is the one maximizing a weighted SLV. Here we view SLV as an importance score, and we want to maximize a quantity which takes into account the importance of the slices being received, weighted by their probability of being received. In this way, the mapping/coding optimization would involve a calculation involving the SLV and channel characteristics, but would not require multiple channel realizations and multiple decodings of the video source.

When the channel coding optimization is based on the SLV, and instantaneous CSI is available at the receiver, the problem formulation is the following. This formulation is provided for whole-sequence optimization, but it can be easily extended to the GOP-by-GOP optimization. Using $B_{\text {tot }}$ to denote the total number of bits which can be transmitted in each RB, we aim to determine the best RCPC code rate profile. Consider $N$ i.i.d. subbands, each with $M$ subcarriers, and packet size for the $j$ th subcarrier equal to $l_{j}$ bits before channel coding using $\mathrm{RCPC} / \mathrm{CRC}$ codes. The constraint on the bit budget/packet can be written as

$$
\sum_{j=1}^{N_{\mathrm{t}}} \frac{l_{j}+B_{c r c}}{R_{j}} \leq B_{t o t}
$$

where $B_{c r c}$ is the bit budget allocated in each packet for the CRC codes, $R_{j}$ is the channel code rate of the RCPC on the $j$ th subcarrier, and $B_{t o t}$ is the total bit budget of the RB. The slices, ordered by decreasing SLV, are characterized by the pair $\left(V_{k}, L_{k}\right)$. To describe the allocation process, we introduce a mapping function $\boldsymbol{\alpha}_{j}$ which allows us to know which slice has been allocated to the $j$ th subcarrier. In particular, $\boldsymbol{\alpha}_{j}=\left[a_{1}^{(j)} a_{2}^{(j)} \ldots a_{\left(N_{\mathrm{F}} \times N_{\mathrm{s}}\right)}^{(j)}\right]^{T}$, with $j=1, \ldots, N_{t}$, and $a_{k}^{(j)}=1$ if the $k$ th slice is allocated to the $j$ th subcarrier, and $a_{k}^{(j)}=0$ otherwise. For Scenario $\boldsymbol{B}$, the most important slices are allocated to the most reliable subbands. This means that the mapping function depends on the instantaneous SNR experienced by the RB, i.e. $\boldsymbol{\alpha}_{j}=\boldsymbol{\alpha}_{j}(\boldsymbol{\gamma}), \forall j \in\left[1, N_{\mathrm{t}}\right]$, where $\boldsymbol{\gamma}=\left[\gamma_{1}, \ldots, \gamma_{N_{t}}\right]$ is the SNR vector and $\gamma_{j}$ is the instantaneous SNR experienced by the $j$ th subcarrier. Note that the mapping function $\boldsymbol{\alpha}_{j}$ depends not only on $\gamma_{j}$, but also on the SNR vector, since the mapping function compares the instantaneous SNR of the $j$ th subcarrier with the instantaneous SNRs of the other subcarriers. So, the bit budget constraint in (1) can be written as

$$
\sum_{j=1}^{N_{t}} \frac{\boldsymbol{L} \cdot \boldsymbol{\alpha}_{j}(\boldsymbol{\gamma}, \boldsymbol{r})+B_{c r c}}{R_{j}} \leq B_{t o t}=L_{\mathrm{p}} \times N_{\mathrm{t}}
$$

where $\boldsymbol{L}=\left[L_{1}, L_{2}, \ldots, L_{N_{\mathrm{F}} \times N_{\mathrm{s}}}\right]$.

Considering the constraint in (2), for the whole-sequence optimization, the best RCPC profile is given by $\boldsymbol{r}^{\star}(\bar{\gamma})$, where

$$
\boldsymbol{r}^{\star}(\bar{\gamma})=\arg \max _{\boldsymbol{r}}\{\mathcal{W} \mathcal{S}(\bar{\gamma}, \boldsymbol{r})\}
$$

and $\mathcal{W S}(\bar{\gamma}, \boldsymbol{r})$ is the weighted SLV for the video sequence, for a given mean SNR $\bar{\gamma}$ and a given UEP profile $r$. We use $N_{\text {gop }}$ to denote the number of GOPs in the video sequence, and $\overline{W S}_{l}(\gamma, \boldsymbol{r})$ to denote the weighted SLV score for the $l$ th GOP of the video. Given the vector of instantaneous SNRs per subcarrier $\gamma$ and the imposed UEP profile $\boldsymbol{r}, \overline{W S}_{l}(\gamma, \boldsymbol{r})$ is defined by

$$
\begin{aligned}
& \overline{W S}_{l}(\gamma, \boldsymbol{r})=\sum_{j=1}^{N_{t}} \boldsymbol{V} \cdot \boldsymbol{\alpha}_{j}(\boldsymbol{\gamma}, \boldsymbol{r}) \cdot\left[1-p_{j}\left(\gamma_{j}, R_{j}\right)\right] \\
& \forall l \in\left[1, \ldots, N_{\text {gop }}\right]
\end{aligned}
$$

where $\boldsymbol{V}=\left[V_{1}, V_{2}, \ldots, V_{N_{\mathrm{F}} \times N_{\mathrm{s}}}\right], \boldsymbol{\gamma}=\left[\gamma_{1}, \ldots, \gamma_{N_{t}}\right]$, and $p_{j}\left(\gamma_{j}, R_{j}\right)$ is the probability of losing the $j$ th subcarrier, which experiences an instantaneous SNR of $\gamma_{j}$ and which has been protected with an RCPC code of rate $R_{j}$. The decision of which RCPC rate to adopt in a given subcarrier depends on the mapping function $\alpha_{j}(\gamma, r)$.

Averaging $\overline{W S}_{l}(\gamma, \boldsymbol{r})$ over the fade vector $\gamma$, we obtain $\mathcal{W S}_{l}(\bar{\gamma})$, which is the weighted visibility score for the $l$ th 
TABLE II

VALUES OF $p(\gamma, R)$, THAT IS THE PACKET LOSS RATE ON A CONDITIONAL AWGN CHANNEL WITH SNR EQUAL TO $\gamma$ AND RCPC CODE RATE EQUAL TO $R$.

\begin{tabular}{|c||c|c|c|c|c|c|c|}
\cline { 2 - 8 } & $\frac{8}{8}$ & $\frac{8}{9}$ & $\frac{8}{10}$ & $\frac{8}{12}$ & $\frac{8}{16}$ & $\frac{8}{20}$ & $\frac{8}{24}$ \\
\hline \hline 2 & 1 & 1 & 1 & 1 & 0.89 & 0.12 & $5 \times 10^{-3}$ \\
\hline 4 & 1 & 1 & 1 & 0.90 & 0.03 & $2 \times 10^{-4}$ & $4.8 \times 10^{-5}$ \\
\hline 6 & 1 & 0.99 & 0.54 & 0.04 & $1.1 \times 10^{-4}$ & $<10^{-6}$ & $<10^{-6}$ \\
\hline 8 & 1 & 0.06 & $6.3 \times 10^{-3}$ & $1.5 \times 10^{-4}$ & $<10^{-6}$ & $<10^{-6}$ & $<10^{-6}$ \\
\hline 10 & 0.98 & $2.5 \times 10^{-4}$ & $7.5 \times 10^{-5}$ & $<10^{-6}$ & $<10^{-6}$ & $<10^{-6}$ & $<10^{-6}$ \\
\hline
\end{tabular}

GOP, for a specific mean SNR $\bar{\gamma}$ and UEP profile $r$. Then, the optimization problem in (3) can be formulated as

$$
\begin{aligned}
& \boldsymbol{r}^{\star}(\bar{\gamma})=\arg \max _{\boldsymbol{r}}\left\{\frac{1}{N_{\text {gop }}} \sum_{l=1}^{N_{\text {gop }}} \mathcal{W} \mathcal{S}_{l}(\bar{\gamma})\right\} \\
& =\arg \max _{\boldsymbol{r}}\left\{\frac{1}{N_{\text {gop }}} \sum_{l=1}^{N_{\text {gop }}} \mathbb{E}_{\boldsymbol{\gamma}}\left\{\sum_{j=1}^{N_{t}} \boldsymbol{V} \cdot \boldsymbol{\alpha}_{j}(\boldsymbol{\gamma}, \boldsymbol{r}) \cdot\left[1-p_{j}\left(\gamma_{j}, R_{j}\right)\right]\right\}\right.
\end{aligned}
$$

given the overall bit budget constraint in (2).

\section{RESUlts}

We carried out simulations on videos of 10 s duration, coded at $R=600 \mathrm{kbps}$ using the H.264/AVC JM codec with SIF resolution $(352 \times 240)$, and with Motion-Compensated Error Concealment (MCEC) as used in [20], implemented in the decoder. For brevity, we provide results for two sequences: "LowMot", and "HighMot". "LowMot" is an almost static video, while "HighMot" has higher motion and several scene changes. We used the IBBP encoding structure with I-frames every 24 frames. There are $N_{\mathrm{t}}=128 \mathrm{OFDM}$ subcarriers in total. The RCPC codes of rates $R_{\text {rcpc }}=$ $\left\{\frac{8}{8}, \frac{8}{9}, \frac{8}{10}, \frac{8}{12}, \frac{8}{16}, \frac{8}{20}, \frac{8}{24}\right\}$ were obtained by puncturing an $R_{c}=1 / 3$ mother code with $K=7, p=8$ and generator polynomials $(133,165,171)_{\text {octal }}$ with the puncturing table given in [21]. QPSK modulation is considered and a slow, block fading channel is assumed. Thus, each subcarrier $j$ will experience a channel fading gain $\gamma_{j}$ that is constant for the whole packet duration. This means that the experienced PLR is equal to the one experienced by a conditional AWGN channel with $\gamma_{j}$ and $R_{j}$ as SNR and RCPC code rate, respectively. In Table II, values of the PLR are provided for several values of $\gamma$ and for the set of RCPC rates considered in our simulations. To evaluate the conditional PLR provided in the table, for each given $\gamma$ and channel code rate, we simulate several channel realizations. We selected the number of these realizations such that PLR values greater than $10^{-6}$ can be evaluated with high precision. The packet size after the RCPC/CRC coding was set equal to 588 bytes, i.e., $L_{\mathrm{m}}=(588 \cdot 4)$ QPSK modulated symbols, such that $L_{\mathrm{m}} \times T_{\mathrm{s}} \approx 24 / 30 \mathrm{~s}$ (to respect the constraint of $30 \mathrm{fps}$ ), where $T_{\mathrm{s}}$ is the symbol period. Due to the imposed constraint, the packet length after the RCPC encoding cannot be greater than $L_{\mathrm{m}}$ modulated symbols. However, for some subcarriers, the number of total bits allocated might be lower than the number of bits that the subcarrier can support. In this case, we use zero padding, in order to have all packets with the same length after the channel coding. Results are provided in terms of the VQM.
For comparison, we consider two baseline algorithms: Sequential and Random. In both of these, we assume that slice importance is not known, and so no packet is more important than another. Thus, EEP is considered for the RCPC coding.

The Sequential algorithm sequentially allocates the slices of each frame to the RB. This means that the first slice of the first frame of the considered GOP will be allocated to the first subcarrier. When no more information bits are available in the first subcarrier, the algorithm starts allocating the current frame to the next subcarrier. Once the slices of the first frame of the GOP are allocated, the second frame is considered. The Random algorithm allocates each slice of the GOP to a random position of the RB.

For the visibility-based model in Scenarios $\boldsymbol{A}$ and $\boldsymbol{C}$, we used 6 visibility groups for the slices (i.e., $K_{v}=6$ ) and considered all possible combinations of RCPC code rates for the 6 groups. In the plots which have RCPC code rate on the Xaxis, the $\mathrm{x}$-coordinate of each plotted value represents the EEP code rate for the random and sequential methods, as well as for the visibility-based Scenario $\boldsymbol{B}$ which also uses EEP. However, for the visibility-based methods in the remaining scenarios which use UEP, the $\mathrm{x}$-coordinate represents the average rate (that is, it is the ratio of the number of information bits to the total bits for the whole sequence). Since a slow fading scenario is considered, we take $f_{n d}=10^{-4}$ throughout this paper, where $f_{n d}$ is the normalized Doppler spread (i.e., its inverse is the coherence time of the channel, expressed in units of symbols). This choice means that there is no time diversity.

In the following figures, we illustrate the proposed channel coding optimization under different fading environments, and the gain that it can achieve in terms of VQM score, when compared to baseline algorithms. We begin by studying the three considered scenarios, providing results for the sequential, the random and the visibility-based algorithm, for different mean SNRs, for both the cases of GOP-by-GOP optimization and whole-sequence optimization. The first results are for the "HighMot" sequence, when the optimization algorithm is aimed at minimizing the VQM score. Then we show how the visibility-based method performs for different video sequences. We show the optimization based on the weighted visibility score for Scenario $\boldsymbol{B}$, and show the optimized results for different SNRs, different numbers of correlated subcarriers, and numbers of total subcarriers.

We first compare the visibility-based and the baseline algorithms for whole sequence optimization. Fig. 5 depicts the VQM vs. the mean RCPC rate when "HighMot" is transmitted over a system with $\mathrm{SNR}=16 \mathrm{~dB}$ and $(N, M)=(32,4)$. The diversity order experienced by the system in the frequency 


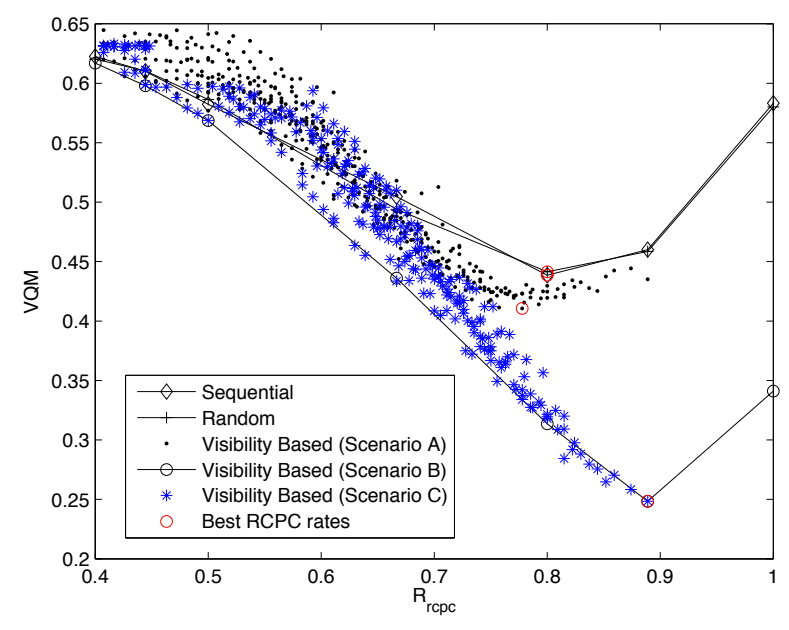

Fig. 5. VQM vs. $R_{r c p c}$ for both visibility-based and baseline algorithms optimized for the whole sequence, for systems with $\mathrm{SNR}=16 \mathrm{~dB}$, $(N, M)=(32,4)$. "HighMot" video is considered.

domain is $\mathcal{D}_{\mathrm{f}}=4$. The visibility-based algorithms were tested for all three scenarios. We observe that the best RCPC profiles for the visibility-based algorithms (the best is the one that produces the lowest VQM value) lead to VQM scores which are better (lower) than the best VQM provided by the sequential or random methods. For Scenario $\boldsymbol{A}$, this means that, even if no instantaneous CSI is assumed in the optimization algorithm, there is a UEP level able to outperform the baseline algorithms. In the literature, a VQM gain of 0.1 is considered to be a significant improvement, and the gain in Fig. 5 is about 0.04 , reached with the best UEP rate vector $\boldsymbol{r}^{*}=[8 / 188 / 128 / 98 / 98 / 98 / 9]$. Note that Scenario A with EEP results in the sequential algorithm. This means that the best VQM achieved by the Scenario A is lower than or equal to the best VQM of the sequential algorithm. However, among all the possible UEP profiles, some of them can lead to a VQM worse than the one achieved by the EEP (i.e., the sequential algorithm). For example, a UEP profile which includes excessive protection for the most important slices can force too many of the unimportant slices to be dropped, resulting in worse performance than EEP. However, the best UEP profile always outperforms the EEP. This behavior is shown in the figure for mean RCPC rates lower than 0.7. Taking into account the instantaneous CSI in the algorithm (Scenarios $\boldsymbol{B}$ and $\boldsymbol{C}$ ), the gain experienced by the proposed method is about 0.2 in VQM score, compared to the baselines. Both Scenarios $\boldsymbol{B}$ and $\boldsymbol{C}$ achieve the same best VQM value, which means that the best UEP FEC profile for the system happens to reduce to EEP (i.e., $r^{*}=[8 / 98 / 98 / 98 / 98 / 98 / 9]$ ). This can be understood by the fact that the instantaneous CSI used in the optimization results in unequal protection to the transmitted slices, and no additional UEP level in the channel coding is required. Moreover, as expected, the case with instantaneous CSI outperforms Scenario $\boldsymbol{A}$ (where only coherence bandwidth information is used), with a noticeable gain of 0.17 in VQM score. The best UEP profile also corresponds to a higher average RCPC rate, and this leads to a reduction in discarded slices.

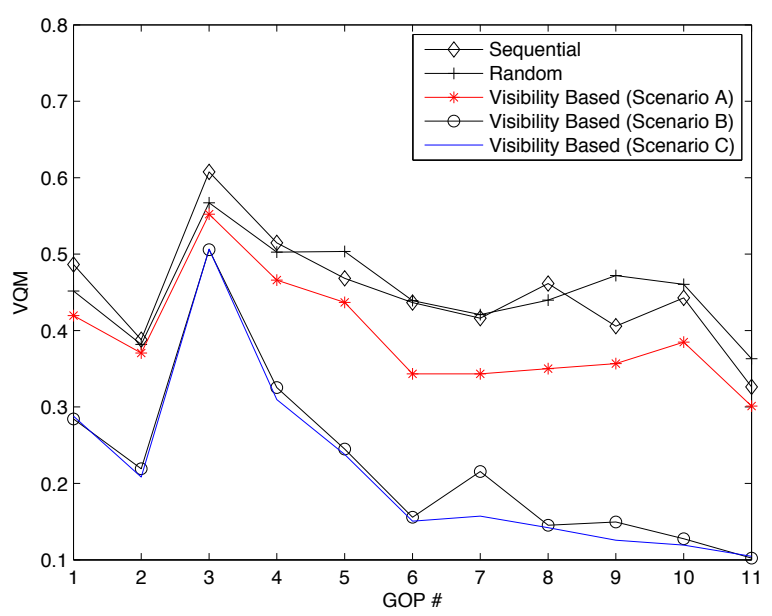

Fig. 6. Best VQM for each GOP of the "HighMot" sequence for visibilitybased and sequential algorithms optimized GOP-by-GOP, for systems with $(N, M)=(32,4)$ and $\mathrm{SNR}=16 \mathrm{~dB}$.

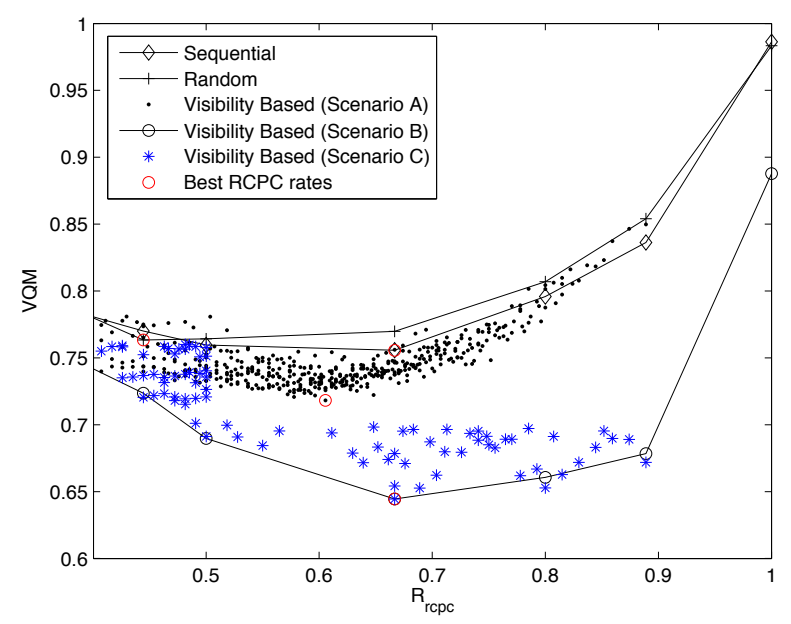

Fig. 7. VQM vs. $R_{\text {rcpc }}$ for both visibility-based and baseline algorithms optimized for the whole sequence, for systems with $\mathrm{SNR}=8 \mathrm{~dB},(N, M)=$ $(32,4)$. "HighMot" video is considered.

We now provide results when the RCPC profile is optimized GOP-by-GOP. Fig. 6 compares the best VQM for each GOP of "HighMot" achieved from the sequential, the random and the visibility-based algorithm (all scenarios) for the same system parameters of the previous figure, i.e., $\mathrm{SNR}=16 \mathrm{~dB}$ and $(N, M)=(32,4)$. As already observed, the proposed algorithm achieves VQM values lower (better) than the ones provided by the baseline algorithms, for all three scenarios. However, here the improvement for some GOPs is very much larger than those in the earlier figure. The visibility-based algorithm in Scenario $\boldsymbol{A}$ outperforms the baseline ones by more than 0.11 in VQM score (GOP 6), although the gain was only 0.04 in VQM score when the RCPC profile was optimized for the whole sequence. As in the previous figure, knowing instantaneous CSI again produces an improvement in performance, when compared to both baseline algorithms and to Scenario $\boldsymbol{A}$. Moreover, it should be noticed that, in the GOP-by-GOP optimization, Scenario $\boldsymbol{C}$ outperforms $\boldsymbol{B}$. 


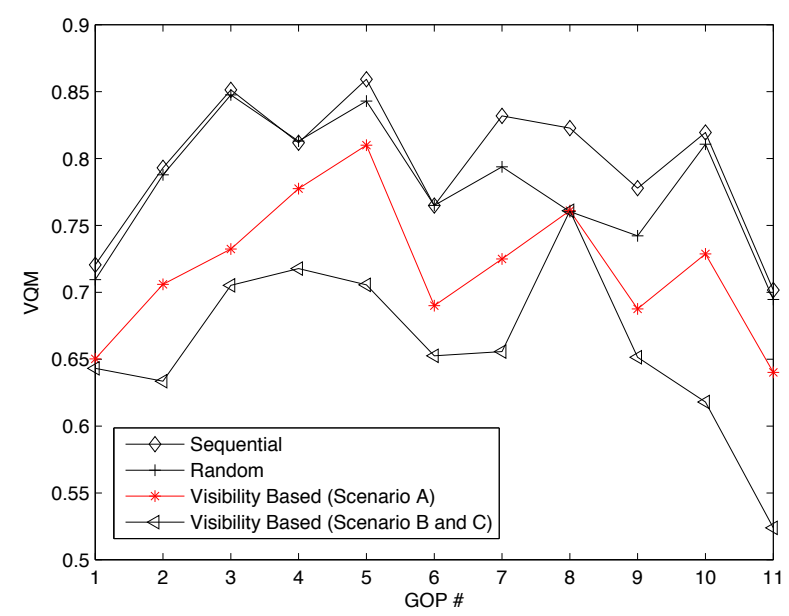

Fig. 8. Best VQM for each GOP of the "HighMot" sequence for visibilitybased and sequential algorithms optimized for the whole sequence, for systems with $(N, M)=(32,4)$ and $\mathrm{SNR}=8 \mathrm{~dB}$.

For example, for the 7 th GOP, Scenario $C$ achieves a VQM of 0.15 , whereas Scenarios $\boldsymbol{A}$ and $\boldsymbol{B}$ achieve scores of 0.34 and 0.21 , respectively. So, for the GOP-by-GOP optimization, UEP optimization improves the performance, even if an unequal level of protection has already been achieved by exploiting knowledge of instantaneous CSI through subcarrier ordering.

Fig. 7 provides the VQM score vs. the mean RCPC code rate when "HighMot" is transmitted over a system with $\mathrm{SNR}=8 \mathrm{~dB}, f_{n d}=10^{-4}$, and $(N, M)=(32,4)$. Compared to the system in Fig. 5, the orders of diversity are the same, while the mean SNR is reduced. This reduction of reliability leads to an increase in the FEC level of the best RCPC code rate for the visibility-based methods. For Scenario $\boldsymbol{A}$, the best FEC profile for the visibility-based algorithm is $\boldsymbol{r}^{*}=[8 / 248 / 168 / 128 / 128 / 128 / 10]$, which leads to a gain in terms of VQM score of 0.03. Due to the low SNR value, the most visually important groups $\Lambda_{i}$ are more heavily protected than they are in the $16 \mathrm{~dB}$ case. This increasing FEC level in the RB keeps the slice loss rate due to channel losses roughly the same as it was for the system with mean $\mathrm{SNR}=16 \mathrm{~dB}$, at the expense of an increase in the number of low-priority slices being discarded prior to transmission. The remaining scenarios experience an EEP of 8/12 as the best FEC profile, which leads to a gain of 0.11 in VQM score, compared to the baseline ones, and a gain of 0.07 with respect to Scenario $\boldsymbol{A}$.

Rather than providing results of the whole sequence optimization in terms of mean VQM, in Fig. 8, the VQM score for each GOP is provided for the "HighMot" sequence for $(N, M)=(32,4), f_{n d}=10^{-4}$, and SNR $=8 \mathrm{~dB}$. As expected, for all the GOPs the visibility algorithms outperform the baseline ones, and Scenario $\boldsymbol{A}$ leads to a VQM score per GOP greater than Scenarios $\boldsymbol{B}$ and $\boldsymbol{C}$ for almost all the GOPs. Moreover, even in these poor channel conditions, where the average improvement for the whole sequence is limited for all the scenarios, the gain of the visibility-based algorithm over the sequential algorithm, for some individual GOPs, is significant, i.e., the gain is up to $0.12(0.2)$ in VQM score

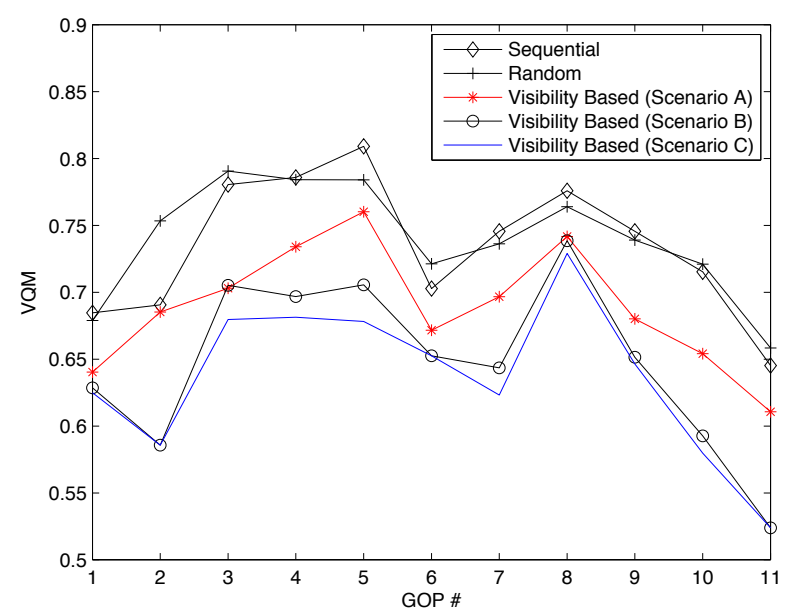

Fig. 9. Best VQM for each GOP of the "HighMot" sequence for visibilitybased and sequential algorithms optimized GOP-by-GOP, for systems with $(N, M)=(32,4)$ and $\mathrm{SNR}=8 \mathrm{~dB}$.

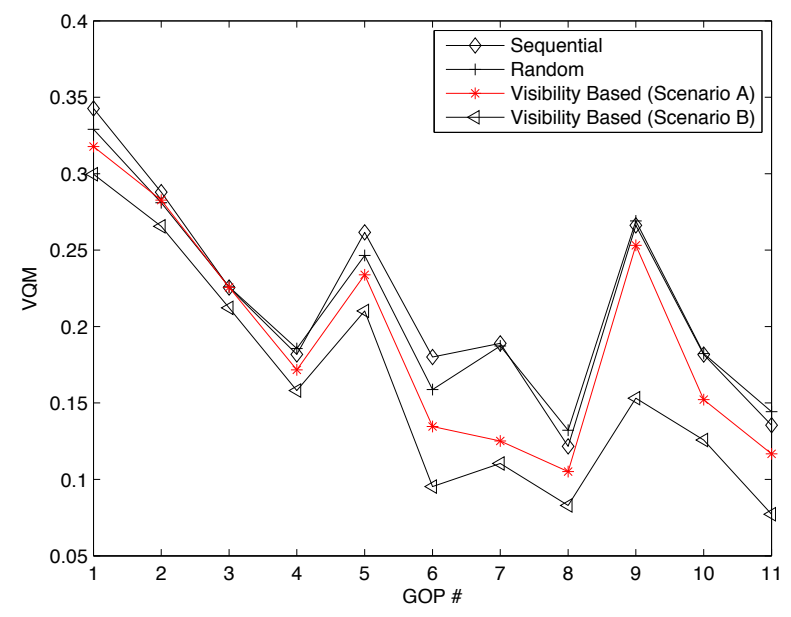

Fig. 10. Best VQM for each GOP of the "LowMot" sequences for visibilitybased and sequential algorithms optimized GOP-by-GOP, for systems with $(N, M)=(32,4)$ and $\mathrm{SNR}=16 \mathrm{~dB}$.

for Scenario $\boldsymbol{A}(\boldsymbol{B}$ and $\boldsymbol{C})$. For the GOP-by-GOP optimization (Fig. 9), the gain of the proposed algorithm is substantial, and Scenario $C$ using both UEP and subcarrier ordering achieves a VQM better (lower) than all the other algorithms.

Fig. 10 depicts the best VQM for each GOP of "LowMot" for $(N, M)=(32,4)$ and SNR $=16 \mathrm{~dB}$. Since, in the whole-sequence optimization, Scenarios $\boldsymbol{B}$ and $\boldsymbol{C}$ led to close results, for clarity in Fig. 10, we study Scenarios $\boldsymbol{A}$ and $\boldsymbol{B}$ only, compared to the sequential algorithm. As for the "HighMot" sequence, the visibility-based algorithms achieve VQM lower (better) than the sequential method, and Scenario $\boldsymbol{B}$ outperforms Scenario $\boldsymbol{A}$. However, for the quasi-static sequence "LowMot", which has few visually important slices, the gain is reduced for most of the GOPs.

After comparing the possible algorithms and scenarios, we now provide results for the case in which the channel code rate is optimized based on the weighted visibility rather than the VQM score. With this aim, we consider the whole sequence 


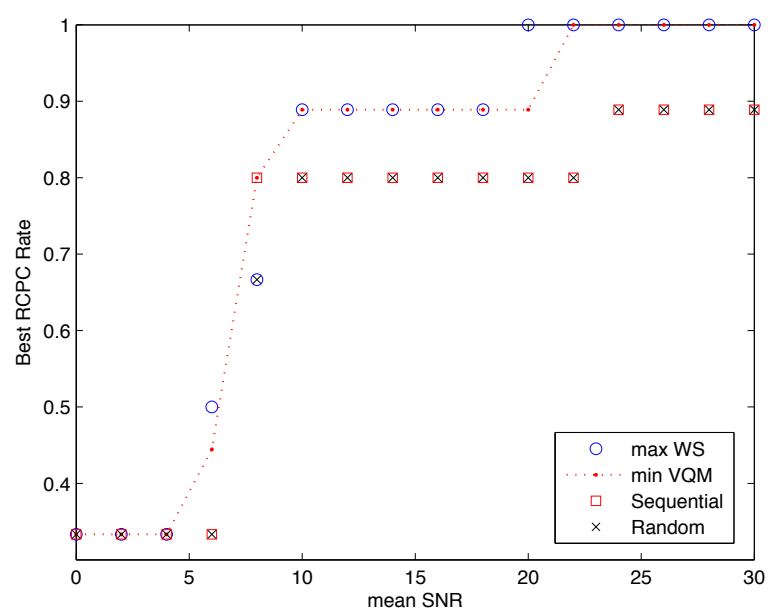

Fig. 11. Best RCPC rates for baseline algorithms and the visibility based algorithm (Scenario $\boldsymbol{B}$ ), aimed at optimizing both the weighted visiblity score and the VQM of the whole video sequence. "HighMot" is considered over systems with $(N, M)=(32,4)$ and variable mean SNRs.

optimization for Scenario $\boldsymbol{B}$, which has been selected due to its simplicity (EEP only is required) and effectiveness. Fig. 11 depicts the best RCPC code rate vs. the mean SNR for the visibility-based algorithm Scenario $\boldsymbol{B}$ (optimized based on both the VQM and the weighted visibility), and the baseline ones (optimized based on the VQM score). From the figure, a close match between the two optimizing methods can be observed. Except for $\bar{\gamma}=6$ and $8 \mathrm{~dB}$, the two optimization techniques lead to the same optimized design. This means that, rather than evaluating the VQM score for each RCPC channel code rate, we can simply evaluate the weighted visibility score for each RCPC configuration and select the best channel code rate. Note that the VQM evaluation requires simulated transmission and decoding of each RB, while the weighted visibility can be evaluated from (5), as detailed in the previous section. We can also see in the figure that, for almost all the mean SNR values, the best channel code rate for the visibilitybased algorithm in Scenario $\boldsymbol{B}$ is greater than the one which achieves the best VQM value in the baseline algorithms. This means that the proposed optimization in Scenario $\boldsymbol{B}$ allows the system to substantially reduce the slice discarding.

In Fig. 12, the best VQM for "HighMot" is provided as a function of $\bar{\gamma}$ for systems with $f_{n d}=10^{-4}$, and $(N, M)=$ $(32,4)$, when Scenario $\boldsymbol{B}$ is considered. For Scenario $\boldsymbol{B}$, we provide two optimization techniques: one based on minimizing the VQM score and one based on maximizing the weighted visibility. Note that for each $\bar{\gamma}$ value, we provided the best VQM optimized over the whole video sequence. As expected, the general behavior (common to all the algorithms) is that the VQM decreases with increasing mean SNR (i.e., with increasing channel reliability). More important, for all the considered mean SNRs, Scenario $\boldsymbol{B}$ outperforms the baseline algorithms, and the gain is up to 0.19 in VQM score (for $\bar{\gamma}=14 \mathrm{~dB}$ ). It is worth noting that, for the comparison of Scenario $\boldsymbol{B}$ optimized based on both the VQM and the one based on the weighted visibility in Fig. 11, we observed that the channel code rate selected as the best differs only

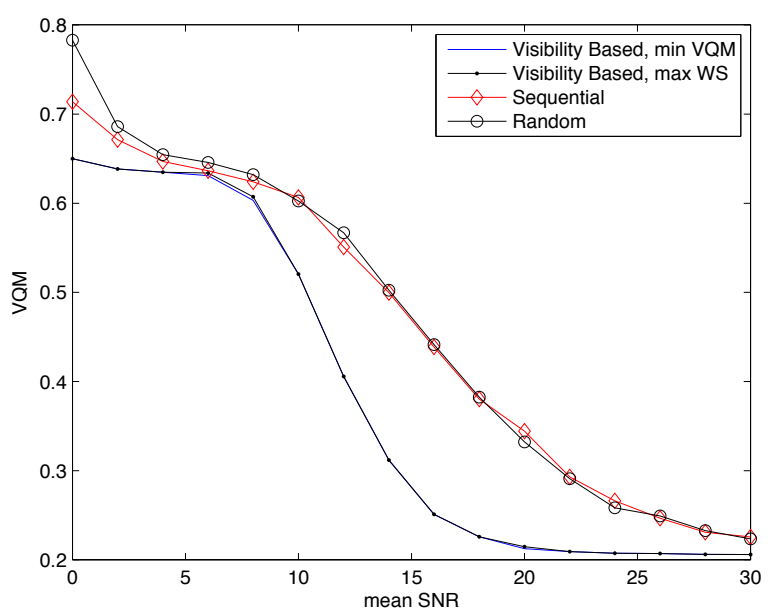

Fig. 12. VQM vs. $\bar{\gamma}$ for both visibility-based and baseline algorithms optimized for the whole sequence, for systems with $(N, M)=(32,4)$. "HighMot" video and Scenario $\boldsymbol{B}$ are considered.

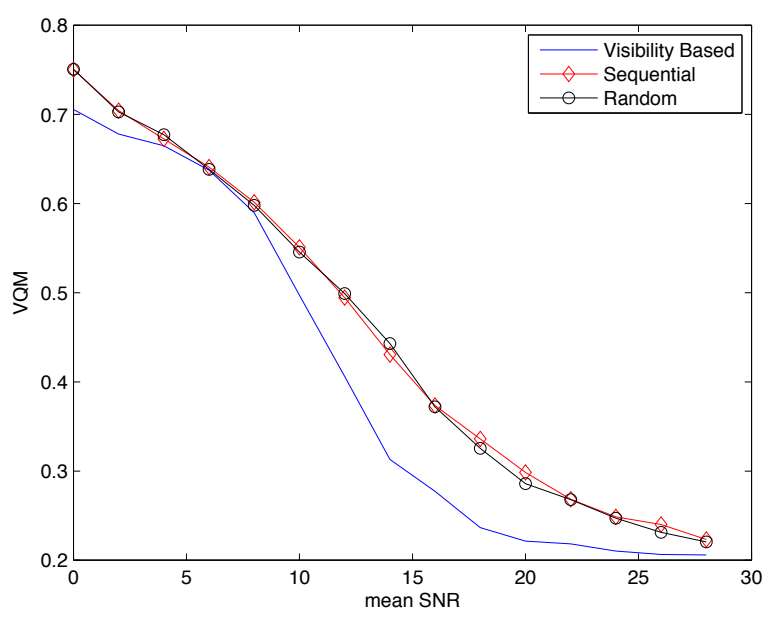

Fig. 13. VQM vs. $\bar{\gamma}$ for both visibility-based and baseline algorithms optimized for the whole sequence for systems with $(N, M)=(8,16)$. "HighMot" video and Scenario $\boldsymbol{B}$ are considered.

at $\bar{\gamma}=6$ and $8 \mathrm{~dB}$. In Fig. 12, this difference in the optimization algorithms can be converted into a VQM score gap. In particular, at $\bar{\gamma}=6$ and $8 \mathrm{~dB}$, the algorithm based on the weighted visibility is outperformed by the one based on the VQM by only 0.005 in VQM score.

We now provide the performance of the proposed algorithm when the optimization is aimed at maximizing the weighted visibility score. We consider the case of a variable number of independent subbands, and we compare the visibility-based algorithm for Scenario $\boldsymbol{B}$ with the baselines. Fig. 13 depicts the system performance when $(N, M)=(8,16)$ and "HighMot" is used for transmission. From the figure, it can be observed that, even reducing the number of independent subbands (which would represent in our model the number of degrees of freedom we can exploit), the visibility-based optimization in Scenario $\boldsymbol{B}$, when compared to the baseline algorithms, still achieves a large gain in terms of VQM. When only 2 independent channels are considered (Fig. 14), as expected, 


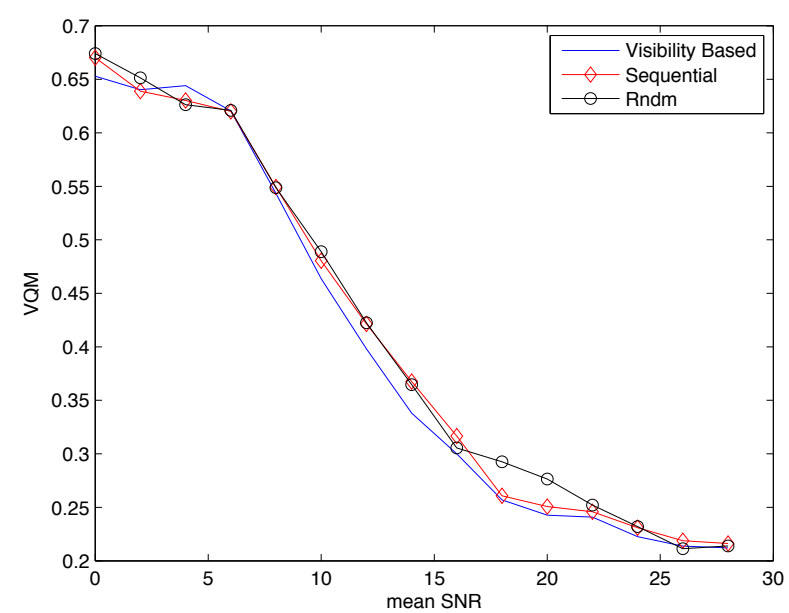

Fig. 14. VQM vs. $\bar{\gamma}$ for both visibility-based and baseline algorithms optimized for the whole sequence for systems with $(N, M)=(2,64)$. "HighMot" video and Scenario $\boldsymbol{B}$ are considered.

due to the limited number of degrees of freedom offered by the channel, the algorithms lead to almost the same performance.

Finally, in Fig. 15, rather than having the number of subcarriers constant and equal to 128, the VQM as a function of the total number of subcarriers is provided for systems with $M=4$ and $\mathrm{SNR}=12 \mathrm{~dB}$, for the "HighMot" sequence and Scenario $\boldsymbol{B}$. Observing the algorithm performance as a function of the system bandwidth, we see that the visibilitybased method significantly outperforms the sequential one most of the time. In particular, to reach a given VQM value, Scenario $\boldsymbol{B}$ requires fewer subcarriers (i.e., smaller bandwidth) compared to the sequential case. For example, a VQM value of 0.4 is reached with the system consisting of 128 subcarriers for the visibility-based algorithm, whereas 278 subcarriers are required for the sequential algorithm, and the capacity gain increases for lower VQM scores.

\section{CONCLUSIONS}

We studied the optimization of channel coding in a 2-D time-frequency resource block of an OFDM system, aimed at maximizing the quality of experience when non-scalable compressed video sequences are transmitted. We used a network-based slice loss visibility (SLV) model to estimate the visual importance of slices to be transmitted over a wireless channel, and to provide the best level of protection to the video slices, opportunistically mapped within the 2D RB. We created three versions of the proposed algorithm, for three different scenarios, characterized by a different level of CSI available in the optimization process, and by different levels of complexity. In all cases, the results demonstrated that the proposed methods outperform the baseline algorithms considered in this paper. In poor channel conditions, due to the high packet loss rates and/or the large number of slices that need to be discarded in order to fit the bitstream within the resource block, the gain of the proposed algorithm is almost negligible, but it increases with the improvement in channel conditions. When instantaneous CSI is available from a feedback channel, the proposed algorithm provides a

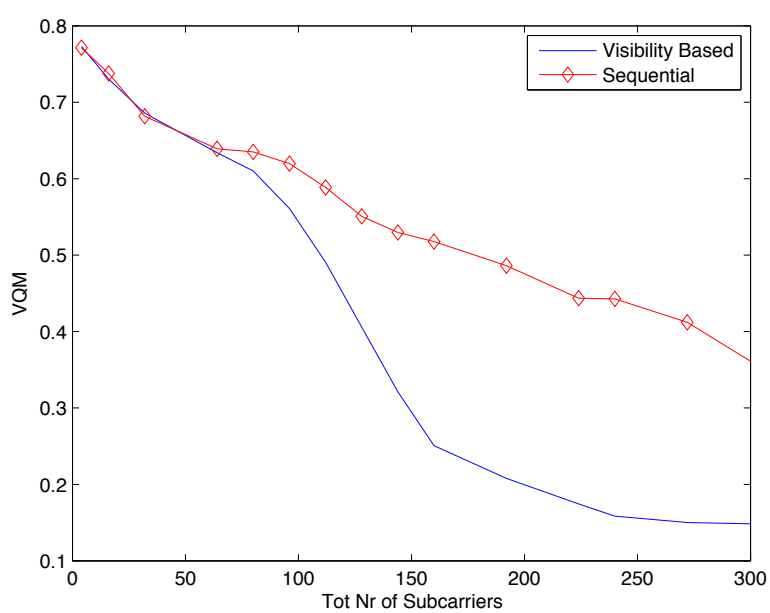

Fig. 15. VQM vs. number of total subcarriers for both visibility-based and baseline algorithms optimized for the whole sequence for systems with $M=4$. "HighMot" video and Scenario $\boldsymbol{B}$ are considered.

considerable improvement in the system performance (up to 0.2 in VQM score), demonstrating the validity of the SLV model in the optimization process. We also showed that, for the parameter values used in these simulations, when instantaneous CSI is available and the FEC profile is optimized for the entire video sequence, an EEP profile is selected as the best profile. However, when the channel coding is optimized GOP-by-GOP, UEP substantially enhances the performance, at the expense of increasing complexity. It was also illustrated that the proposed technique is especially useful for video sequences with medium-high motion content, which means in video sequences for which a substantial portion of slices are visually important. Lastly, in order to simplify the optimization process, the channel coding scheme can be optimized for the weighted visibility score. Results demonstrated the reliability of this measure in several physical environments.

\section{REFERENCES}

[1] S. Zhao, Y. Zhang, and L. Gui, "Optimal resource allocation for video delivery over MIMO OFDM wireless systems," in Proc. GlobeCom, Dec. 2010.

[2] S.-H. Chang, P. Cosman, and L. Milstein, "Performance analysis of nchannel symmetric FEC-based multiple description coding for OFDM networks," IEEE Trans. Image Process., vol. 20, no. 4, pp. 1061 -1076, April 2011.

[3] N. Thomos, J. Chakareski, and P. Frossard, "Prioritized video streaming through randomized network coding," IEEE Trans. Multimedia, vol. 13, no. 4, pp. 776-787, Aug. 2011.

[4] R. Serral-Graci, E. Cerqueira, M. Curado, M. Yannuzzi, E. Monteiro, and X. Masip-Bruin, "An overview of quality of experience measurement challenges for video applications in IP networks," in Wired/Wireless Internet Communications, ser. Lecture Notes in Computer Science, 2010, vol. 6074, pp. 252-263.

[5] A. R. Reibman, V. A. Vaishampayan, and Y. Sermadevi, "Quality monitoring of video over a packet network," IEEE Trans. Multimedia, vol. 6, no. 2, pp. 327 - 334, April 2004.

[6] F. M. S. Cho and W. A. Pearlman, "Robust image transmission using a new joint source channel coding algorithm and dual adaptive OFDM," in Proc. GlobeCom, San Jose, CA, U.S.A., Jan. 2004.

[7] S. S. Arslan, P. C. Cosman, and L. B. Milstein, "Progressive source transmissions using joint source-channel coding and hierarchical modulation in packetized networks," in Proc. GlobeCom, Nov. 2009. 
[8] L. Toni, Y. S. Chan, P. C. Cosman, and L. B. Milstein, "Channel coding for progressive images in a 2-D time-frequency OFDM block with channel estimation errors," IEEE Trans. Image Process., vol. 18, no. 11, pp. $2476-2490$, Nov. 2009.

[9] N. Thomos, S. Argyropoulos, N. V. Boulgouris, and M. G. Strintzis, "Robust transmission of H.264/AVC streams using adaptive group slicing and unequal error protection," EURASIP J. Appl. Signal Process., vol. 2006, pp. 120-120, Jan. 2006.

[10] T.-L. Lin and P. C. Cosman, "Efficient optimal RCPC code rate allocation with packet discarding for pre-encoded compressed video," IEEE Signal Process. Lett., vol. 17, no. 5, pp. 505 -508, May 2010.

[11] Y. J. Liang, J. G. Apostolopoulos, and B. Girod, "Analysis of packet loss for compressed video: Effect of burst losses and correlation between error frames," IEEE Trans. Circuits Syst. Video Technol., vol. 18, no. 7, pp. $861-874$, July 2008.

[12] Y.-L. Chang, T.-L. Lin, and P. Cosman, "Network-based IP packet loss importance model for h.264 sd videos," in 18th International Packet Video Workshop (PV), 2010, Dec. 2010, pp. $178-185$.

[13] B. Chen and J. Francis, Multimedia Performance Evaluation. AT\&T Technical Memorandum, May 2003.

[14] G. W. Cermak, Videoconferencing Service Quality as a function of bandwidth, latency, and packet loss. T1A1.3/2003-026, May 2003.

[15] C. J. Hughes, M. Ghanbari, D. E. Pearson, V. Seferidis, and J. Xiong, "Modeling and subjective assessment of cell discard in ATM video," IEEE Trans. Image Process., vol. 2, no. 2, pp. 212 -222, April 1993.

[16] S. Kanumuri, P. C. Cosman, A. R. Reibman, and V. A. Vaishampayan, "Modeling packet-loss visibility in MPEG-2 video," IEEE Trans. Multimedia, vol. 8, no. 2, pp. 341-355, 2006.

[17] T.-L. Lin, S. Kanumuri, Y. Zhi, D. Poole, P. C. Cosman, and A. R. Reibman, "A versatile model for packet loss visibility and its application to packet prioritization," IEEE Trans. Image Process., vol. 19, no. 3, pp. $722-735$, Mar. 2010.

[18] R. J. McEliece and W. E. Stark, "Channels with block interference," IEEE Trans. Inf. Theory, vol. 30, no. 1, pp. 44-53, Jan. 1984.

[19] "VQM software," in [Online]. Available: http://www.its.bldrdoc.gov/n3/video/vqmsoftware.htm.

[20] S. Kanumuri, S. G. Subramanian, P. C. Cosman, and A. R. Reibman, "Packet-loss visibility in H.264 videos using a reduced reference method," in Proc. ICIP, Oct. 2006.

[21] P. Frenger, P. Orten, T. Ottosson, and A. Svensson, "Multi-rate convolutional codes, technical report no. 21," Department of Information Theory, Chalmers University of Technology, Tech. Rep., Apr. 1998.

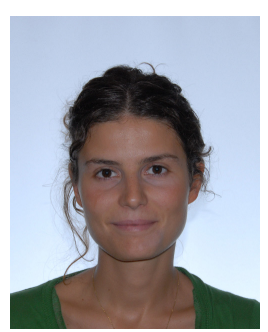

Laura Toni (S'06-M'09) received the M.S. degree (with honors) in electrical engineering and the Ph.D. degree in electronics, computer science and telecommunications from the University of Bologna, Italy, in 2005 and 2009, respectively.

In 2005, she joined the Department of Electronics, Informatics and Systems at the University of Bologna, to develop her research activity in the area of wireless communications. During 2007, she was a visiting scholar at the University of California at San Diego, CA, working on video processing over wireless systems. Since 2009, she has been a frequent visitor to the UCSD, working on joint source and channel coding for wireless communication systems.

From June 2009 to November 2011, she worked at the Tele-Robotics and Application (TERA) department at the Italian Institute of Technology (IIT) as a Post-doctoral Fellow. Since November 2011, she has been a Postdoctoral fellow in the Electrical and Computer Engineering department at the University of California, San Diego. Her research interests are in the areas of image and video processing, wireless communications, and underwater communications.

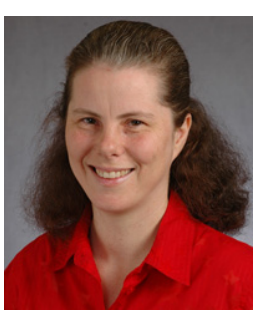

Pamela C. Cosman (S'88-M'93-SM'00-F'08) obtained her B.S. with Honor in Electrical Engineering from the California Institute of Technology in 1987, and her M.S. and Ph.D. in Electrical Engineering from Stanford University in 1989 and 1993, respectively.

She was an NSF postdoctoral fellow at Stanford University and a Visiting Professor at the University of Minnesota during 1993-1995. In 1995, she joined the faculty of the department of Electrical and Computer Engineering at the University of California, San Diego, where she is currently a Professor and Vice Chair. She was the Director of the Center for Wireless Communications from 2006 to 2008. Her research interests are in the areas of image and video compression and processing, and wireless communications.

Dr. Cosman is the recipient of the ECE Departmental Graduate Teaching Award, a Career Award from the National Science Foundation, a Powell Faculty Fellowship, and a Globecom 2008 Best Paper Award. She was a guest editor of the June 2000 special issue of the IEEE Journal on Selected Areas in Communications on "Error-resilient image and video coding," and was the Technical Program Chair of the 1998 Information Theory Workshop in San Diego. She was an associate editor of the IEEE Communications Letters (1998-2001), and an associate editor of the IEEE Signal Processing Letters (2001-2005). She was the Editor-in-Chief (2006-2009) as well as a Senior Editor (2003-2005, 2010-present) of the IEEE Journal on Selected Areas in Communications. She is a member of Tau Beta Pi and Sigma Xi.

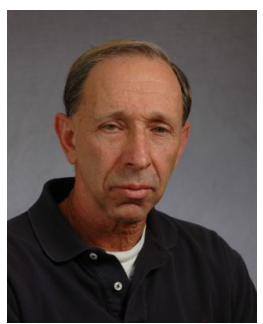

Laurence B. Milstein (S'66-M'68-SM'77-F85) received the B.E.E. degree from the City College of New York, New York, in 1964, and the M.S. and $\mathrm{Ph} . \mathrm{D}$. degrees in electrical engineering from the Polytechnic Institute of Brooklyn, Brooklyn, NY, in 1966 and 1968, respectively.

From 1968 to 1974 , he was with the Space and Communications Group, Hughes Aircraft Company, and from 1974 to 1976 , he was a member of the Department of Electrical and Systems Engineering, Rensselaer Polytechnic Institute, Troy, NY. Since 1976, he has been with the Department of Electrical and Computer Engineering, University of California, San Diego, San Diego, where he is currently the Ericsson Professor of Wireless Communications Access Techniques and former Department Chair, working in the area of digital communication theory with special emphasis on spread-spectrum communication systems. He has also been a consultant to both government and industry in the areas of radar and communications.

Dr. Milstein was an Associate Editor for Communication Theory for the IEEE TRANSACTIONS ON COMMUNICATIONS, an Associate Editor for Book Reviews for the IEEE TRANSACTIONS ON INFORMATION THEORY, an Associate Technical Editor for the IEEE Communications Magazine, and the Editor-in-Chief of the IEEE JOURNAL ON SELECTED AREAS IN COMMUNICATIONS. He was the Vice President for Technical Affairs in 1990 and 1991 of the IEEE Communications Society and was a former chair of the IEEE Fellows Selection Committee. He was a recipient of the 1998 Military Communications Conference Long Term Technical Achievement Award, an Academic Senate 1999 UCSD Distinguished Teaching Award, an IEEE Third Millennium Medal in 2000, the 2000 IEEE Communication Society Armstrong Technical Achievement Award, and various prize paper awards, including the 2002 MILCOM Fred Ellersick Award. He was also the recipient of the IEEE Communication Theory Technical Committee (CTTC) service Award in 2009, and the CTTC Achievement Award in 2012. 